\title{
Application of Accelerometer in Table Tennis Action Recognition
}

\author{
Yin Chunyu
}

Northwest University, Xi’an 710069, China

Keywords: Acceleration sensor; Table tennis; Action recognition

\begin{abstract}
As an important technology in human-machine interaction, action recognition is one of the research hotspots in the pattern recognition. Accelerometer is a kind of wearable sensor. It is characterized by low energy consumption, small volume and low cost, which is widely used. At present, the action recognition process based on acceleration sensing is mainly divided into 3 steps: feature extraction, feature selection and recognition algorithm. In this paper, a recognition algorithm based on three-dimensional acceleration signal is proposed to identify different types of strokes according to the motion characteristics of people playing table tennis. The data used are collected from the accelerometer tied to the wrist and collected in the training process of table tennis. The threshold value of acceleration signal is judged by fixed threshold method. And the key characteristics of signal energy and gesture length are extracted.
\end{abstract}

\section{Introduction}

Table tennis is a world popular ball game. China has always been in the leading position throughout the world, while China's research on table tennis robots is still in its initial stage[1].

As an important technology of human-machine interaction, action recognition has always been one of the hotspots in the field of pattern recognition[2]. There are two main directions in motion recognition researches [3]: motion recognition based on visual sensor and motion recognition based on wearable sensors. The action recognition technology based on acceleration sensor is the latter, because the acceleration sensor has the characteristics of low energy consumption, small volume, low cost, so it is widely used. At present, the action recognition process based on acceleration sensing is mainly divided into three steps [4]: feat extraction, feature selection and recognition algorithm. Feature extraction is mainly in two aspects: the physical characteristics of signals and the characteristics of human motion. In terms of physical features, wavelet transform and fast Fourier transform are used to extract frequency and energy information[5]. In terms of motion characteristics, the average values, entropy and eigenvalues of signal vector magnitude (SVM) are extracted. Based on the three-dimensional acceleration sensor, this paper designs the recognition system of the stroke motion[6]. Through the acquisition, processing and analysis of the acceleration data, the hand stroke motion based on the three-dimensional acceleration sensor is obtained. It has a high recognition rate for batting types and a careful classification of batting types[7].

\section{Introduction of acceleration sensor}

Acceleration sensor can measure acceleration, which is usually composed of mass blocks, dampers, elastic elements, sensitive elements and adaptive circuits. During the acceleration process, the accelerometer is obtained by measuring the inertial force of the mass block and using Newton's second law. According to the different sensor sensors, the common acceleration sensors include capacitive, inductive, strain, piezoresistive, piezoelectric, etc. [8]

Piezoelectric accelerometer is also called piezoelectric accelerometer. The principle of a piezoelectric accelerometer is the piezoelectric effect of a piezoelectric ceramic or quartz crystal. When the accelerometer is vibrated, the force of the mass on the piezoelectric element is also changed. When the measured vibration frequency is much lower than the natural frequency of the accelerometer, the change of force is directly proportional to the acceleration measured. 
Based on the world's leading MEMS silicon micromachining technology, the piezoresistive accelerometer has the characteristics of small size, low power consumption and so on. It is easy to integrate in all kinds of analog and digital circuits. Capacitive accelerometer is a capacitance sensor based on capacitance principle. Servo accelerometer is a closed loop test system, which is featured by good dynamic performance, large dynamic range and good linearity.

\section{Research method of action recognition}

Motion recognition is a direction in the field of pattern recognition research. With the popularization and miniaturization of computers, as well as the development of artificial intelligence, action recognition has been developed rapidly and has been widely used in all walks of life, such as physical game, intelligent monitoring. Action recognition mainly deals with analyzing the behavior pattern of an object by way of computer modeling. Common algorithms of action recognition include decision tree algorithm, hidden Markoff model algorithm, support vector machine algorithm, artificial neural network algorithm and so on[9].

Artificial neural network, also known as the connection model, is a mathematical model simulating human brain structure for information processing. Template matching recognition algorithm mainly includes dynamic programming and dynamic time warping. The template matching recognition algorithm is widely used in the speech recognition and action recognition. However, in the process of template matching, each template in the template library needs to be matched and compared, so the computation is large and the execution efficiency is low, which is also the main factor restricting the development of the template[10].

\section{Data acquisition}

The hardware device of this experiment is mainly divided into two parts: data acquisition module and data receiving module. The data acquisition module is made up of three-dimensional acceleration sensor and wireless data transmission device, and the data receiving and processing module is composed of data receiving device and PC machine.

In order to obtain the acceleration information in the table tennis ball, the experimental data acquisition module is fixed on the wrist, which is used to collect the acceleration signal of gestures. When the acceleration sensor senses the action of the hand, the analog acceleration signal can be obtained. Digital signals are obtained through A/D conversion. They are transmitted to the data receiving device through the wireless local area network. The PC machine saves the data read from the receiving device to the database. The computer extracts features of acceleration data and performs gesture recognition analysis. Its schematic diagram is shown in Figure 1.

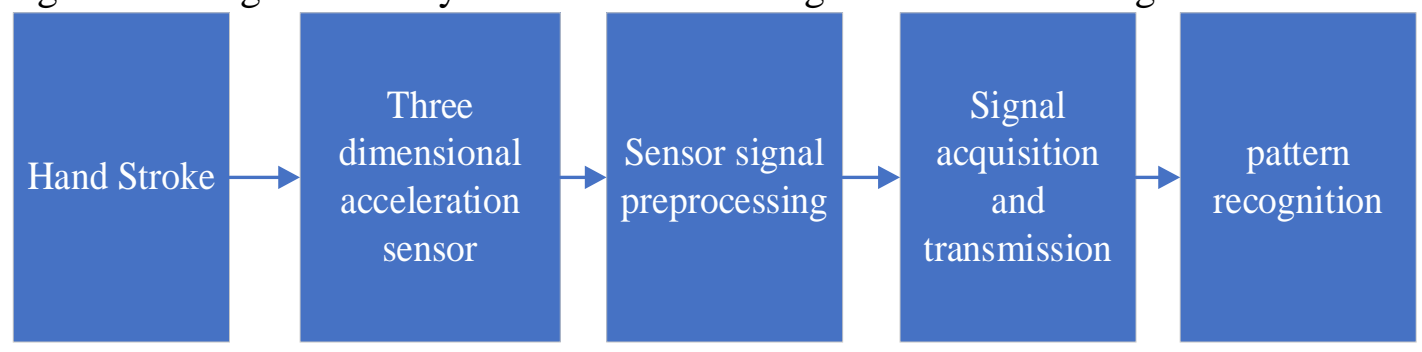

Figure 1 Table tennis action recognition principle map.

Whether the feature selection of action is appropriate or not, it is directly related to the real-time performance and accuracy of classification algorithm. Through analyzing the sample data, the characteristics of signal energy, gesture length, signal peak and valley value and motion characteristics of the signal are chosen to analyze the data.

\section{Characteristic action recognition classification}

$\mathrm{K}-\mathrm{NN}$ algorithm is a basic classification and regression algorithm. Decision tree algorithm is a 
method to approximate the value of discrete function, and it is a typical classification method[11]. In this paper, a decision tree classification algorithm incorporating K-NN is proposed. First, the gesture energy and gestures length are used to remove the interference, and then the K-NN algorithm is used to divide the big classes of the push, the ball and the attack. Finally, the left and right movements are identified by the wave through characteristics of the wave peak. When the KNN's decision tree algorithm is used to classify the data, although the algorithm increases relative to the K-NN algorithm in the use, the recognition accuracy is $98.2 \%$, which is obviously higher than the classification result only using the K-NN algorithm.

\section{Conclusions}

This paper mainly introduces the recognition system of table tennis hitting action based on accelerometer. Compared to the research based on the modelling of image processing and kinematics principle, this paper tries to combine the acceleration sensor with the person in the table tennis. It is very good to study the different types of batting movements in table tennis by studying the acceleration signal data.

Acceleration data are obtained by accelerometer fixed on the wrist. K-NN's decision tree algorithm is used to classify actions. It has the characteristics of high recognition rate and fine action classification. Because of the characteristics of the selection, there may be redundant features. The efficiency of the algorithm needs to be further improved, and feature selection should be considered.

\section{Acknowledgements}

The authors wish to acknowledge the collaborative funding support from the Australian Research Council, Access Online Pty Ltd and Allette Systems Ltd. Under grant no. C4991-7612. In particular we wish to thank Vassiliki Elliott, Ross Jeffery, Louise Scott, Lucila Carvalho, John D'Ambra, Nick Carr and Marcus Carr for their contributions to this research project.

We also wish to acknowledge the valuable contributions of the numerous companies and individuals who participated in the industry interviews and surveys.

\section{References}

[1] DING Wenwen,LIU Kai,XU Biao,CHENG Fei.Skeleton-Based Human Action Recognition via Screw Matrices[J].Chinese Journal of Electronics,2017,26(04):790-796.

[2] Shuyang Li,Wei Liang,Qun Zhang.Human-Object Interaction Recognition Based on Modeling Context[J].Journal of Beijing Institute of Technology,2017,26(02):215-222.

[3] Xijia Liu,Xiaoming Tao,Ning Ge.Fast Remote-Sensing Image Registration Using Priori Information and Robust Feature Extraction[J].Tsinghua Science and Technology,2016,21(05):552560.

[4] TANG Wei,JING Bo,HUANG YiFeng,SHENG ZengJin.Feature extraction for latent fault detection and failure modes classification of board-level package under vibration loadings.[J].Science China(Technological Sciences),2015,58(11):1905-1914.

[5] Deng Sen,Jing Bo,Sheng Sheng,Huang Yifeng,Zhou Hongliang.Impulse feature extraction method for machinery fault detection using fusion sparse coding and online dictionary learning[J].Chinese Journal of Aeronautics,2015,28(02):488-498.

[6] GAO Huizhong,LIANG Lin,CHEN Xiaoguang,XU Guanghua.Feature Extraction and Recognition for Rolling Element Bearing Fault Utilizing Short-Time Fourier Transform and Nonnegative Matrix Factorization[J].Chinese Journal of Mechanical Engineering,2015,28(01):96-105.

[7] Xiao-Fei Ji,Qian-Qian Wu,Zhao-Jie Ju,Yang-Yang Wang.Study of Human Action Recognition Based on Improved Spatio-temporal Features[J].International Journal of Automation \&amp; 
Computing,2014,11(05):500-509.

[8] LIU CuiWei,PEI MingTao,WU XinXiao,KONG Yu,JIA YunDe.Learning a discriminative midlevel feature for action recognition[J].Science China(Information Sciences),2014,57(05):195-207.

[9] ZHANG WeiLong,LIU LeiBo,YIN ShouYi,ZHOU RenYan,CAI ShanShan,WEI ShaoJun. An efficient VLSI architecture of speeded-up robust feature extraction for high resolution and high frame rate video[J].Science China(Information Sciences),2013,56(07):136-149.

[10] LUO Yuan,WU Cai-ming,ZHANG Yi.Facial expression feature extraction using hybrid PCA and LBP[J].The Journal of China Universities of Posts and Telecommunications,2013,20(02):120124.

[11] M. Marsadek,A. Mohamed.Risk based security assessment of power system using generalized regression neural network with feature extraction[J].Journal of Central South University, 2013, 20(02):466-479. 